\title{
LARGE EDDY SIMULATION OF ACOUSTIC CHARACTERISTICS OF A SUBSONIC JET OUTFLOWING FROM CONICAL NOZZLE
}

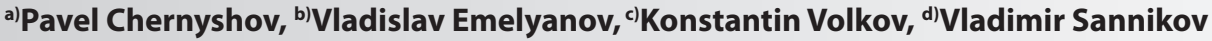 \\ a)Baltic State Technical University «VOENMEH» named after D.F. Ustinov, St. Petersburg, Russia, pashachp8@gmail.com \\ ${ }^{b)}$ Baltic State Technical University «VOENMEH» named after D.F. Ustinov, St. Petersburg, Russia, ve5303@mail.ru \\ c)Kingston University, London, United Kingdom, k.volkov@kingston.ac.uk \\ d)Baltic State Technical University «VOENMEH» named after D.F. Ustinov, St. Petersburg, Russia,
}

\begin{abstract}
The calculation of noise generated by a jet of viscous compressible gas flowing out from a conical nozzle is considered. The calculations used the implicit version of the LES (Implicit LES, ILES), in which the role of the subgrid turbulence model performs numerical dissipation used finite-difference scheme. The distributions of the gas-dynamic and acoustic characteristics of the jet upon changing the conditions of its outflow are discussed. The analysis of the modal composition of the received noise is carried out and the correspondence between the features of the received directionality of the noise is determined by its various components and sources. The numerical simulation results are compared with the available experimental and calculated data.
\end{abstract}

Keywords: acoustics, jet, noise, numerical simulation, turbulence

DOI: 10.36336/akustika202139256

\section{INTRODUCTION}

The problem of improving the acoustic perfection of the design of jet engines is one of the priorities in modern aviation. Noise, being a harmful environmental factor, can cause significant harm not only to living organisms, but also to the materials of the aircraft structure. In a certain frequency range, it has a direct effect on human organs and the system of his cranial nerves. One of the major contributors to aircraft engine noise is jet noise.

Supersonic jets are a canonical flow that includes both complex shock wave interactions and turbulent mixing. The jet has three noise sources with different mechanisms of action: turbulent pulsations in the mixing layer, vibration of shock waves, interaction between shock waves and turbulence. At low speeds, only turbulent pulsations contribute.

The results of experimental and numerical studies of supersonic jets and their generation of noise have been reflected in a large number of publications [1-4]. Difficulties in calculating noise are partly related to the impossibility of rigorously distinguishing acoustic density (or pressure) fluctuations against the background of vortex and entropic disturbances present in a turbulent flow. In practice, empirical models of the main characteristics of aerodynamic noise (spectral composition, radiation pattern) are used, obtained on the basis of experimental studies of model and full-scale gas jets on open acoustic stands.

To improve the accuracy of describing turbulent flows in the presence of shock waves, relations are used that limit the growth of turbulence energy and turbulent viscosity near shock waves $[5,6]$. The most common methods for modifying the turbulence model are to develop approximate relations for the terms responsible for the effect of compressibility on turbulence in the equation for the transfer of kinetic energy of turbulence $[7,8]$, as well as in changing the constants of the turbulence model $[9,10]$. Various approaches to the calculation of noise generated by cold and heated subsonic jets are compared in the work [11]. A simplified semi-empirical model for calculating noise is proposed in the work [10]. Turbulent mixing noise consists of two separate sources: noise generated by Mach waves (large-scale structures) and propagating downstream, as well as noise generated by small-scale structures that are dominant in the vicinity of the jet boundary. The gas-dynamic and acoustic characteristics of jets outflowing from conical nozzles are discussed in [12, 13], and self-oscillatory processes in supersonic jets are discussed in [14].

Despite the advances in turbulence modeling and the development of aeroacoustics, accurate calculation of the characteristics of jet stream noise is still a difficult task. There are models of various levels of accuracy for calculating the noise of a jet stream: direct numerical simulation, simulation of large vortices, and acoustic schemes based on the solution of the Reynolds-averaged Navier-Stokes equations. The modern approach for calculating noise is based on eddy-resolving modeling of the flow field in the jet, using Kirchhoff surfaces and the integral form of the wave equation (for example, the FW-H equation) in the far acoustic field outside the jet. For practical use of aeroacoustics methods necessary to optimize use of computing technology that eliminates or minimizes the unnecessary costs of computing resources.

This article considers the calculation of the noise generated by a subsonic jet, and discusses the distributions of the gas-dynamic and acoustic characteristics of the jets when the conditions of their outflow change. The analysis of the modal composition of the received noise is carried out and the correspondence between the features of the received directionality of the noise is determined by its various components and 
sources. The numerical simulation results are compared with the available experimental and calculated data.

\section{NUMERICAL SIMULATION}

\subsection{Computational domain and mesh}

The geometric parameters of the jet outflowing from the conical nozzle are the diameter of the nozzle outlet section $\boldsymbol{d}_{\boldsymbol{a}}$ and the nozzle half-opening angle $\boldsymbol{\theta}_{a}$. An axisymmetric conical nozzle with a nozzle exit diameter $\boldsymbol{d}_{a}=76.20 \mathrm{~mm}$ and a nozzle half-opening angle $\boldsymbol{\theta}_{a}=25^{\circ}$ is used to form the jet. The inner diameter of the cylindrical section is $\boldsymbol{d}_{\boldsymbol{i}}=145.48 \mathrm{~mm}$, and its outer diameter is $\boldsymbol{d}_{\boldsymbol{o}}=185.20 \mathrm{~mm}$.

The computational domain is a rectangle $\left[\mathbf{0}, \boldsymbol{L}_{\boldsymbol{x}}\right] \times\left[\mathbf{0}, \boldsymbol{L}_{\boldsymbol{y}}\right]$. The length of the computational domain is $\mathbf{8 0 d}_{\boldsymbol{a}^{\prime}}$, where $\mathrm{d}$ is the outlet section of the conical nozzle. The distance from the nozzle exit to the front boundary of the surrounding space is $\mathbf{1 0 d}_{a^{\prime}}$ which is equal to the distance from the inlet boundary of the jet stream to the outlet section of the conical nozzle. The distance from the axis of rotation of the nozzle to the upper boundary of the surrounding space is $\mathbf{3 5} \boldsymbol{d}_{\boldsymbol{a}}$.

For the numerical calculation, a hybrid computational grid with block structuring elements is used. In the near flow field, where it is necessary to resolve the largest range of turbulence scales, a block-structured computational grid is built. Close to the nozzle walls, the mesh is refined anisotropically to a step of $2.54 \times 10^{-5} \mathrm{~m}$ at a value of $\mathrm{y}^{+} \sim 1.8$. This makes it possible to calculate the near-wall boundary layer with good accuracy.

Refinement of the mesh near the jet flow region provides adequate resolution of instability waves of the shear layer, shock-wave structure, propagation of acoustic waves in the near field. In the direction of the output boundaries, the computational grid is roughened (the size of large cells is about 25 times the wavelength).

\subsection{Calculation method}

For a complete calculation of aeroacoustics, as a problem that is part of a gas-dynamic calculation, in the near and far fields, it is necessary to discretize the space over the entire region of propagation of acoustic waves to determine the entire range of turbulent pulsations. For most tasks, such an approach can be not only unreasonable, but also impracticable due to the amount of required computational costs.

To circumvent such limitations, hybrid methods have been developed in which the turbulent flow zone and the region in which acoustic waves propagate are separated. In this approach, the near-field flow is calculated using vortex-resolving turbulence models, and the linearized Euler equations are solved in the region of acoustic wave propagation. This approach has some limitations, such as the impossibility of considering many aerocoustic effects that do not arise in the near flow field, which can greatly affect the accuracy of the solution. Nevertheless, this approach allows one to solve with good accuracy most practically significant problems without the use of large computational costs.

The processes of excitation and generation of sound by gas flows are inextricably linked with the general problem of de- scribing turbulent flows. In accordance with the theory of the development of perturbations, turbulence is considered as an essentially nonlinear stage in the evolution of small perturbations, characterized by intense irregular mixing of macroscopic liquid volumes and energy transfer to smaller volumes [15].

There are several main approaches to the numerical modeling of the gas dynamics of turbulent flows: the approach in modeling based on the solution of the Reynolds Averaged Navier Stokes (RANS) equations, the Large Eddy Simulation (LES) method, the direct numerical modeling method (Direct Numerical Simulation, DNS). The direct numerical method in computational gas dynamics involves the solution of non-averaged, three-dimensional, and non-stationary $\mathrm{Na}$ vier-Stokes equations at all turbulence scales, which makes it possible to obtain instantaneous characteristics of a turbulent flow. This approach provides the highest accuracy. In practice, the computational resources required to accomplish the task using DNS are often too large, which significantly narrows the range of tasks that can be solved by this method. RANS is based on solving Reynolds-averaged Navier-Stokes equations. RANS is most widely used in engineering practice, largely due to its small requirements for computing resources. This method introduces the hypothesis of the existence of isotropic turbulence at each point of the flow, due to which this approach simulates the entire spectrum of turbulent pulsations and does not reveal the vortex structure of the flow, which is often extremely important from a practical point of view. LES is performed by solving the full Navier-Stokes equations with the elimination of small-scale turbulence by a filtering operation. In contrast to DNS, in LES, the Navier-Stokes equations are calculated only for large eddies that carry the maximum Reynolds stresses and are directly affected by the boundary conditions. Through this approach LES much less exacting to computing resources, rather than DNS, and provides a much more accurate results than the RANS. Unlike RANS, LES reveals the vortex structure of the flow.

The calculations used the implicit version of the LES (Implicit LES, ILES), in which the role of the subgrid turbulence model performs numerical dissipation used finite-difference scheme. The role of subgrid viscosity is played by the numerical dissipation of the used computational scheme. This is since, when calculating the noise of turbulent jets by the LES method, it is important to provide a realistic description of the transition from laminar to turbulent flow in the mixing layers adjacent to the nozzle edge. This process at high Reynolds numbers occurs quickly, which determines the range of noise frequencies that are resolved within the LES. Resolution of small-scale vortex structures coming from the boundary layer on the nozzle wall and leading to turbulence of the mixing layer turns out to be impossible due to high requirements for computational resources. As a rule, the use of classical (explicit) models of subgrid viscosity leads to a delay in the transition process. One of the ways to eliminate this drawback is based on replacing real turbulent pulsations at the nozzle exit with artificial ones. However, this approach introduces spurious noise that is difficult to filter from the true one. 


\subsection{Results}

Consider the calculation of acoustic noise in the far field from a compressible turbulent jet flowing from a converging nozzle into a flooded space. The main defining parameters of the problem are the Mach number of a fully expanded jet, equal to 0.9 , and the Reynolds number, constructed from the parameters corresponding to the nozzle exit, which is $1.2 \times 106$. The time step is $10^{-7} \mathrm{~s}$ (about $1.8 \times 106$ steps for the entire calculation). The time step is chosen from the Courant condition (CFL 0.8).

At the first stage, the problem is solved in the full computational domain within the RANS solution. In this case, a mesh is used that is sufficient to resolve the boundary layer on the nozzle walls, but coarser in coordinates tangential to the wall than that required in the LES. At the second stage, LES calculation of the jet field is performed. In this case, the distributions of flow parameters obtained at the RANS stage (profiles of pressure and stagnation temperatures, as well as profiles of entry angles) are used as boundary conditions at the nozzle exit. The instantaneous velocity field is shown in Fig. 1.

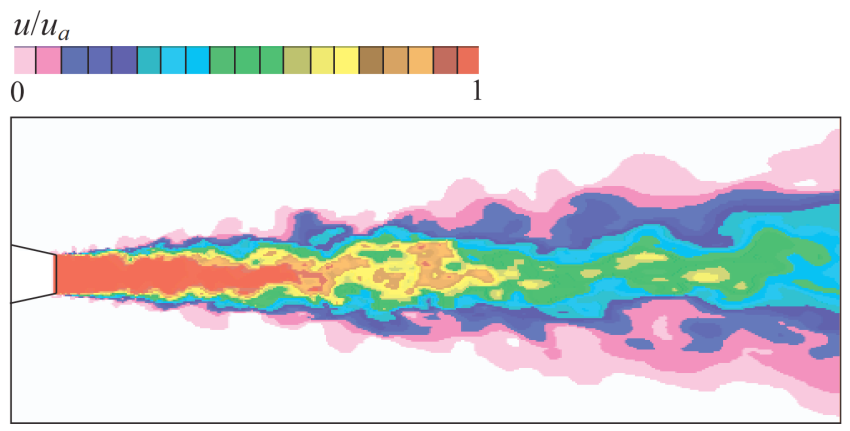

Fig. 1: Instantaneous velocity field after establishment of current

Instantaneous pressure fields are shown in Fig. 2 in two versions corresponding to the choice of gas-dynamic and acoustic scales of pressure change. Low-frequency waves are clearly visible on the pressure distribution on the acoustic scale. The instantaneous field of the derivative of pressure with respect to time is shown in Fig. 3.

The calculation of the sound pressure away from the nozzle is performed using the acoustic analogy method. For this, in the computational domain, a special surface is allocated, covering the jet from the outside, from which the values of the fields of velocity, density and pressure are taken during the calculation. These values are subsequently processed by a special subroutine that calculates the integral $\mathrm{FW}-\mathrm{H}$. The noise level is determined at the points located at a distance $\boldsymbol{r} / \boldsymbol{d}_{\boldsymbol{a}}=98$ from the nozzle exit, where $\boldsymbol{d}_{\boldsymbol{a}}$ is the nozzle exit diameter, in the range of polar angles $20^{\circ}-160^{\circ}$. The obtained data are additionally averaged over 10 azimuth angles. Comparison of one-third-octave far-field noise spectra with experimental data [16] shows Fig. 4. Comparison of the directional patterns of the integral jet noise with the experimental data [16] and [17] is shown in Fig. 5.

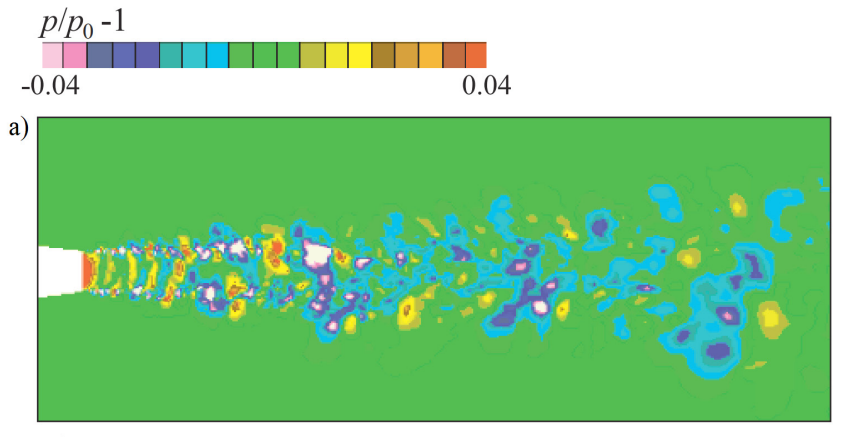

$$
\begin{aligned}
& p / p_{0}-1 \\
& \begin{array}{rl|l|l|l|l|l|l}
\hline \mid & \\
-0.002 & & &
\end{array}
\end{aligned}
$$

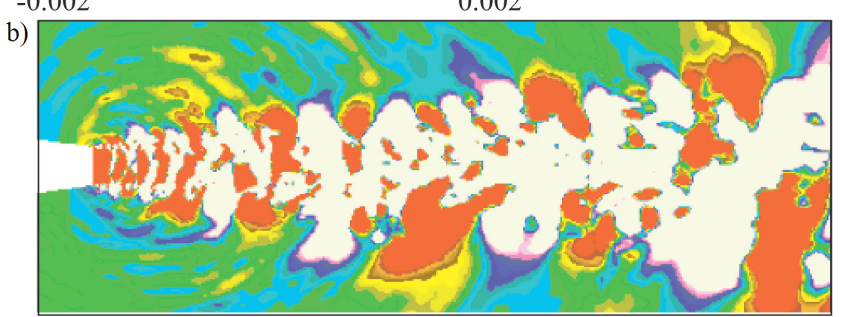

Fig. 2: Pressure fields in the gas-dynamic (a) and acoustic (b) scales

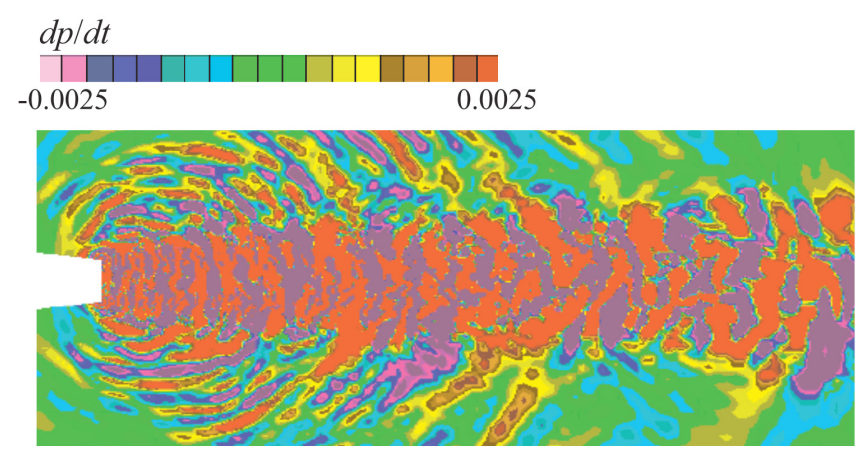

Fig. 3: Field of the derivative of pressure with respect to time after the establishment of the flow

In the range of Strouhal numbers $0.2<\mathrm{St}<1.5$, the spectra are in satisfactory agreement with the experimental data. The spectral energy is distributed evenly with some bias towards high frequencies, while its integral level corresponds to the experimental one.

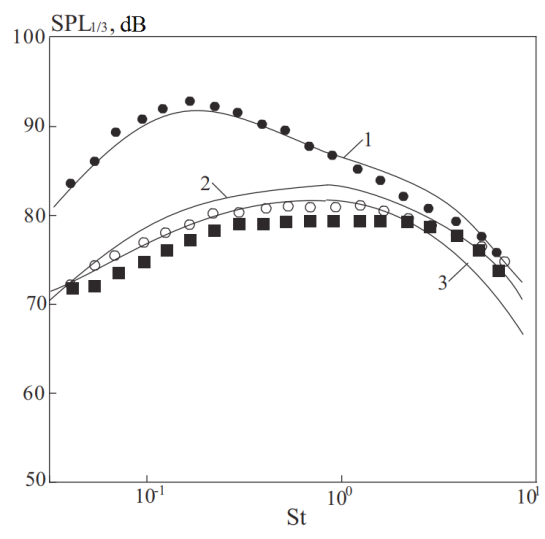

Fig. 4: Comparison of the calculated (continuous lines) one-third-octave far-field noise spectra with experimental data [16] (symbols) at $\theta=30^{\circ}(1, \cdot), 90^{\circ}\left(2,{ }^{\circ}\right), 120^{\circ}(3$, 


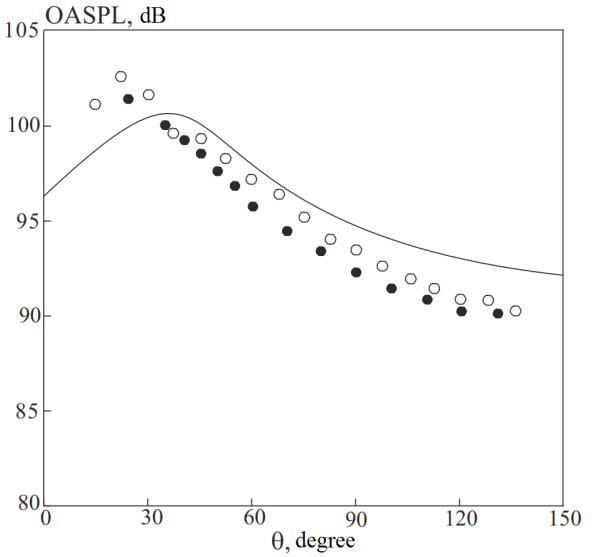

Fig. 5: Comparison of the calculated (continuous line) radiation patterns of the integral noise with experimental data [16] (symbols ${ }^{\circ}$ ) and [17] (symbols •)

The calculation of the distribution (continuous lines) of the integral sound pressure level along the angular coordinate at different Mach numbers at the nozzle exit (subsonic outflow mode) is shown in Fig. 6, which is compared with the experimental data of [18] (symbols) at $\boldsymbol{r} / \mathbf{D}=53$.

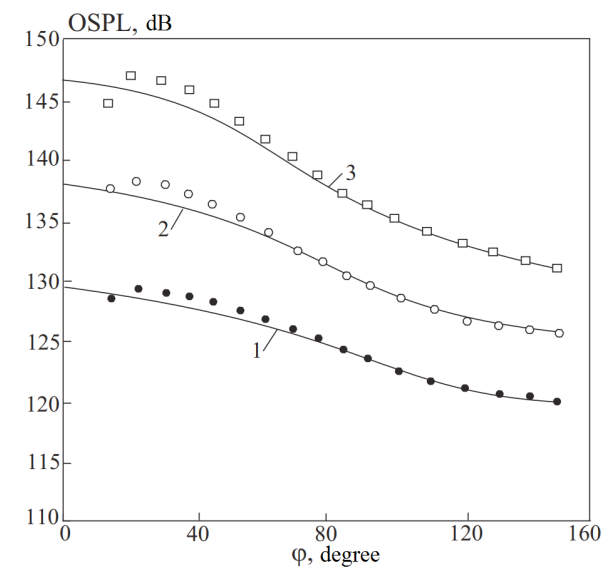

Fig. 6: Distribution of the integral sound pressure level along the angular coordinate at various Mach numbers $M=0.6(1, \cdot)$, $0.75\left(2,{ }^{\circ}\right), 0.9(3$,

The distribution of the sound pressure level over frequencies at different angular coordinates and a fixed Mach number at the nozzle exit $(\boldsymbol{M}=0.75)$ is shown in Fig. 7 in comparison with the experimental data from [18] (symbols).

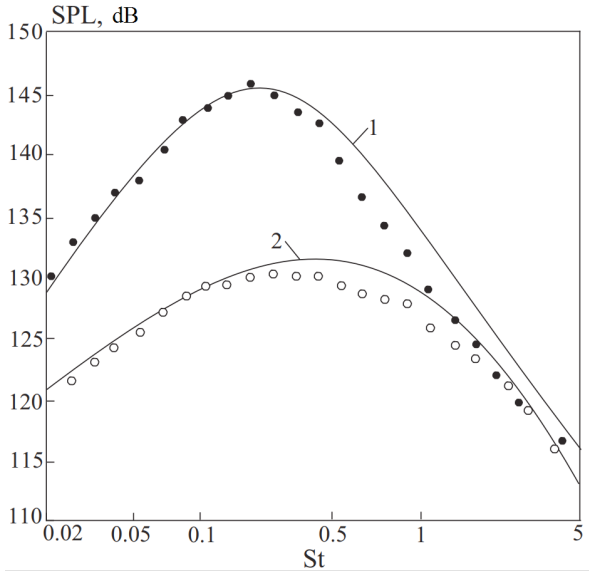

Fig. 7: Distribution of the sound pressure level over frequencies at different angular coordinates $\varphi=30^{\circ}(1, \cdot)$ and $90^{\circ}\left(2,{ }^{\circ}\right)$

\section{CONCLUSION}

Compared to classical gas dynamics, computational aeroacoustics problems are characterized by an increased degree of complexity. One of the reasons is that the characteristic size of acoustic waves in air is several orders of magnitude larger than the size of the pulsations responsible for energy transfer.

Another difficulty is that in many cases the acoustic energy is only a small fraction of the total energy. Due to the significant scatter in the characteristic scales, the correct calculation of the acoustic field seems to be rather difficult for numerical simulation of the generation and propagation of sound.

A numerical study is carried out and the influence of the outflow parameters on the characteristics of the jet outflowing from a conical nozzle, as well as the distribution of parameters along the jet axis is shown. Comparison of the obtained results with experimental data shows that the calculation results reproduce the jet structure rather well, and the local and integral flow parameters are in good agreement with the physical experiment data.

The developed approaches make it possible with satisfactory accuracy to carry out calculations of unsteady three-dimensional turbulent flows of a viscous compressible gas and acoustic processes occurring during the outflow of gas jets from a nozzle into a flooded space, and the use of supercomputer technologies leads to a reduction in the time spent on research. 


\section{REFERENCES}

[1] Raman, G.: Supersonic jet screech: half-century from Powell to the present, Journal of Sound and Vibration, Vol. 225, No. 3., P. 543-571, 1999

[2] Chin, C., Li, M., Harkin, C., Rochwerger, T., Chan, L., Ooi, A.: Investigation of the flow structures in supersonic free and impinging jet flows, Journal of Fluids Engineering, Vol. 135, No. 3, 031202 (12 pages), 2013

[3] Zapryagaev, V., Kiselev, N., Gubanov, D.: Shock-wave structure of supersonic jet flows, Aerospace, Vol. 5, Paper No. 60 (18 pages), 2018

[4] Chernyshov, P., Emelyanov, V., Tsvekov, A., Volkov K.: Large-eddy simulation of noise generated by pulsed supersonic jets, Journal Akustika, Vol. 34, p. 137-141, 2019

[5] Emami, B., Bussman, M., Tran, H.: Application, realizability and shock unsteadiness to k- $\varepsilon$ simulations of under expanded axisymmetric supersonic free jets, Journal of Fluid Engineering, Vol. 132, No. 4, 041104, 2010

[6] Nichols, D.S.: Accounting for shocks in the ke-kw turbulence model, AIAA Paper, No. 2011-3573, 2011

[7] Oh Y.H., Bushnell D.M.: Influence of external disturbances and compressibility on free turbulent mixing, NASA Langley Research Center, NASA SP-347, 1975

[8] Sarkar, S.: Modeling the pressure-dilatation correlation, NASA Report. 1991. No. CR-187566, 1991

[9] Thies, A.T., Tam, C.K.W.: Computation of turbulent axisymmetric and nonaxisymmetric jet flows using the k-epsilon model, AIAA Journal, Vol. 34, No. 2,p. 309-316, 1996

[10] Tam, C.K.W., Auriault, L.: Jet mixing noise from fine-scale turbulence, AIAA Journal, Vol. 37, No. 2,p. 145-153, 1999

[11] Trumner, J., Mundt, C.: Comparison of two different CAA methods for the prediction of far-field noise from heated and unheated jets, Proceedings of the 7th European Conference for Aeronautics and Aerospace Sciences (EUCASS), 3-6 July 2017, Milan, Italy, EUCASS2017-568 (14 pages), 2017

[12] Kim, J.H., Kearney-Fischer, M., Samimy, M., Gogineni, S.: Far-field noise control in supersonic jets from conical and contoured nozzles, Journal of Engineering for Gas Turbines and Power, Vol. 133, 081201 (9 pages), 2011

[13] Alam, M.M.A., Setoguchi, T., Matsuo, S., Kim, H.D.: Nozzle geometry variations on the discharge coefficient, Propulsion and Power Research, Vol. 5, No. 1,p. 22-33, 2016

[14] Emelyanov, V.N., Tsvetkov, A.I., Volkov K.N.: Mechanisms of generation and sources of noise in supersonic jets, Journal Akustika.Vol. 32,p. 144-150, 2019

[15] Volkov, K.N.: Large-eddy simulation of free shear and wall-bounded turbulent flows, Atmospheric Turbulence, Meteorological Modelling and Aerodynamics / P.R. Lang and F.S. Lombargo. Nova Science, p. 505-574, 2010

[16] Viswanathan, K.: Aeroacoustics of hot jets, Journal of Fluid Mechanics, Vol. 516, p. 39-82, 2014

[17] Tanna, H.K.: An experimental study of jet noise. Part I. Turbulent mixing noise, Journal of Sound and Vibration, Vol. 50,p. 405-428, 1977

[18] Bogey, C., Barre, S., Fleury, V., Bailly, C., Juve, D.: Experimental study of the spectral properties of near-field and far-field jet noise, International Journal of Aeroacoustics, Vol. 6. No. 2. p. 73-92, 2007

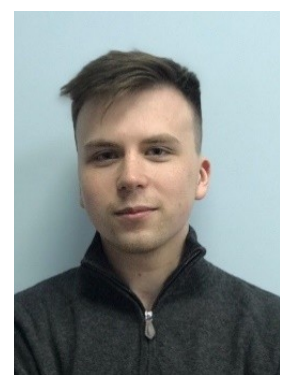

Pavel Chernyshov was born in 1996. Graduate student of Department of Plasma and Gas Dynamics and Heating Engineering of the Baltic State Technical University (BSTU).

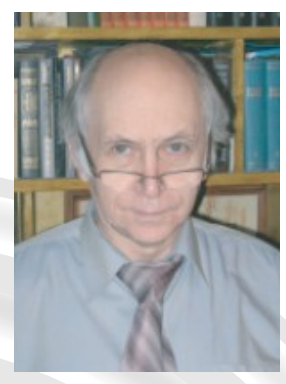

Vladislav Emelyanov was born in 1941. Graduated from the Leningrad Mechanical Institute (LMI) in 1965. Professor, Doctor of Sciences, Head of Department of Plasma and Gas Dynamics and Heating Engineering of BSTU. During his work in BSTU, created a new scientific direction in research of gas dynamics and heat transfer processes in devices of rocket and space engineering, based on combination of physical and mathematical simulation. Author of 180 academic papers and 6 books. Was granted an award of the Honorary Worker of Higher School and medals of Federation of Cosmonautics. Academic adviser of the Russian Academy of Rocket and Artillery Sciences. 

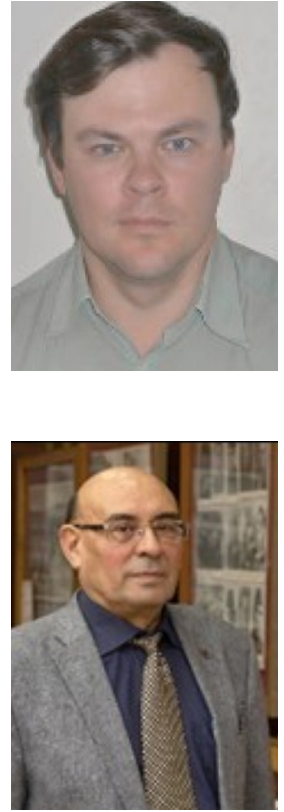

Konstantin Volkov was born in 1973. Graduated from the Baltic State Technical University (BSTU) in 1996 and the Saint Petersburg State University in 1997. Doctor of Philosophy since 1998 and Doctor of Sciences since 2007. Worked in BSTU as a research assistant from 1996 to 2002, from 1998 to 2002 as a lecturer, then a senior lecturer, at the Department of Plasma and Gas Dynamics and Thermal Engineering. Worked in the United Kingdom at the University of Central Lancashire from 2002 to 2004 and University of Surrey from 2004 to 2009. From 2009 works and lectures in the Kingston University in London. During his work in BSTU, took part in development and achievement of models designed for the description of intra-chamber processes in solid rocket motors. Author of over 170 academic papers and 10 books. Member of the Institute of Physics and the Institution of Mechanical Engineers (United Kingdom).

Vladimir Sannikov is a Doctor of Engineering Science, Professor of Environment and Safety chair of the Baltic State Technical University 'VOENMEH'.

He is the author of more than 160 scientific papers, also the coauthor of 15 textbooks and guides, 1 patent. It deals with problems of fluctuation of limited environments, dynamics of technical designs, decrease in level of vibration, level of structural noise from source of various physical nature. The participant of a number of the international conference, seminars in the field of acoustic and ecology which were carried out in St. Petersburg, including the international conference 'NOISE 93', the 4th International Congress of a sound and vibration (1996) and others. 